From the University of Torino, Azienda Ospedaliero-Universitaria (AOU) San Giovanni Battista, Torino; "Seràgnoli" Institute of Hematology, Bologna University School of Medicinew, Bologna University Hospital, Bologna; University of Catania, Catania; AOU di Udine, Udine; Università del Piemonte Orientale Amedeo Avogadro, Novara; "Sapienza" University of Rome; Università Tor Vergata, Ospedale S Eugenio, Rome; Spedali Civili Brescia, Brescia; Ospedali Riuniti, Bergamo; AOU Careggi, Firenze; Università degli Studi di Bari “Aldo Moro," Policlinico, Bari; University of Modena and Reggio Emilia, Modena; Fondazione Istituto di Ricovero e Cura a Carattere Scientifico (IRCCS), Istituto Nazionale dei Tumori; Università degl Studi di Milano, Fondazione IRCCS Cà Granda, Ospedale Maggiore Policlinico, Milano; Ospedale San Bortolo, Vicenza; Azienda Ospedaliera "Bianchi-MelacrinoMorelli," Reggio Calabria; Clinica P.O. Pescara, Pescara; AOU San Martino, Genova; Università di Padova, Ematologia $e$ Immunologia Clinica, Padova; Ospedale Centrale Bolzano, Bolzano; Ospedale Santa Maria delle Croci, Ravenna; AOU Ospedali Riuniti di Ancona, Ancona; and Azienda Ospedaliera di Cosenza, Cosenza, Italy. Submitted July 16, 2010; accepted November 29, 2010; published online ahead of print at www.jco.org on January 31, 2011.

Both A.P. and M.C. contributed equally to this work.

Authors' disclosures of potential conflicts of interest and author contributions are

found at the end of this article.

Clinical Trials repository link available on JCO.org.

Corresponding author: Michele Cavo, MD

"Seràgnoli" Institute of Hematology,

Azienda Ospedaliero-Universitaria S. Orsola

Malpighi, Bologna, Italy; e-mail:

michele.cavo@unibo.it.

C 2011 by American Society of Clinical Oncology

0732-183X/11/2908-986/\$20.00

DOI: 10.1200/JCO 2010.31.6844

\title{
Aspirin, Warfarin, or Enoxaparin Thromboprophylaxis in Patients With Multiple Myeloma Treated With Thalidomide: A Phase III, Open-Label, Randomized Trial
}

Antonio Palumbo, Michele Cavo, Sara Bringhen, Elena Zamagni, Alessandra Romano, Francesca Patriarca Davide Rossi, Fabiana Gentilini, Claudia Crippa, Monica Galli, Chiara Nozzoli, Roberto Ria, Roberto Marasca, Vittorio Montefusco, Luca Baldini, Francesca Elice, Vincenzo Callea, Stefano Pulini, Angelo M. Carella, Renato Zambello, Giulia Benevolo, Valeria Magarotto, Paola Tacchetti, Norbert Pescosta, Claudia Cellini, Claudia Polloni, Andrea Evangelista, Tommaso Caravita, Fortunato Morabito, Massimo Offidani, Patrizia Tosi, and Mario Boccadoro

\section{$\begin{array}{llllllll}\text { A } & \text { B } & \text { S } & \text { T } & \text { R } & \text { A } & \text { C } & \text { T }\end{array}$}

\section{Purpose}

In patients with myeloma, thalidomide significantly improves outcomes but increases the risk of thromboembolic events. In this randomized, open-label, multicenter trial, we compared aspirin (ASA) or fixed low-dose warfarin (WAR) versus low molecular weight heparin (LMWH) for preventing thromboembolism in patients with myeloma treated with thalidomide-based regimens.

\section{Patients and Methods}

A total of 667 patients with previously untreated myeloma who received thalidomide-containing regimens and had no clinical indication or contraindication for a specific antiplatelet or anticoagulant therapy were randomly assigned to receive ASA $(100 \mathrm{mg} / \mathrm{d})$, WAR $(1.25 \mathrm{mg} / \mathrm{d})$, or LMWH (enoxaparin $40 \mathrm{mg} / \mathrm{d}$ ). A composite primary end point included serious thromboembolic events, acute cardiovascular events, or sudden deaths during the first 6 months of treatment.

\section{Results}

Of 659 analyzed patients, $43(6.5 \%)$ had serious thromboembolic events, acute cardiovascular events, or sudden death during the first 6 months $16.4 \%$ in the ASA group, $8.2 \%$ in the WAR group, and $5.0 \%$ in the LMWH group). Compared with LMWH, the absolute differences were $+1.3 \%(95 \% \mathrm{Cl},-3.0 \%$ to $5.7 \% ; P=.544)$ in the ASA group and $+3.2 \%(95 \% \mathrm{Cl},-1.5 \%$ to $7.8 \% ; P=.183$ ) in the WAR group. The risk of thromboembolism was 1.38 times higher in patients treated with thalidomide without bortezomib. Three major $(0.5 \%)$ and 10 minor $(1.5 \%)$ bleeding episodes were recorded.

\section{Conclusion}

In patients with myeloma treated with thalidomide-based regimens, ASA and WAR showed similar efficacy in reducing serious thromboembolic events, acute cardiovascular events, and sudden deaths compared with LMWH, except in elderly patients where WAR showed less efficacy than LMWH.

\section{J Clin Oncol 29:986-993. (C) 2011 by American Society of Clinical Oncology}

\section{INTRODUCTION}

In patients with cancer, the incidence of venous thrombosis is more than $7 \%^{1}$ but remains an underdiagnosed and undertreated condition. ${ }^{2}$ Myeloma is associated with the highest risk of thromboembolism. ${ }^{3}$

Cancer chemotherapy amplifies the prothrombotic effect of cancer cells and damages vessel walls directly. ${ }^{4}$ The combination of melphalan, prednisone, and thalidomide is now a standard of care for the initial treatment of elderly patients with myeloma. ${ }^{5-7}$ However, thalidomide significantly increases the risk of thromboembolism. ${ }^{8-10}$
Small studies have evaluated the clinical benefits of single-agent antithrombotic prophylaxis (aspirin [ASA], warfarin [WAR], or low molecular weight heparin [LMWH]). Results of ASA and lowdose WAR are conflicting. ${ }^{11-14}$ In a meta-analysis of 1,051 patients, the relative risk of thromboembolism in patients treated with thalidomide and LMWH was still 1.54 times higher than in patients not receiving thalidomide. ${ }^{10}$ The American Society of Clinical Oncology guidelines recommend prophylaxis with LMWH or adjusted-dose WAR in patients receiving thalidomide. ${ }^{15}$ At present, no randomized study has directly compared the clinical benefits of ASA, WAR, and LMWH as thromboprophylaxis for 
patients with myeloma treated with thalidomide. This multicenter, randomized, open-label study compared the efficacy and safety of ASA or fixed low-dose WAR with LMWH in preventing thromboembolism in untreated patients with myeloma who received thalidomide-containing regimens.

\section{PATIENTS AND METHODS}

\section{Study Design and Treatment}

This open-label, phase III, randomized study was conducted at 84 centers in Italy during May 2006 to January 2009 as a common substudy of two simultaneous chemotherapy phase III trials using thalidomide-based regimens in previously untreated patients with myeloma. ${ }^{16,17}$ The aim of this study was to compare the effectiveness of ASA and WAR with LMWH as antithrombotic prophylaxis. The study was approved by the institutional review board at each of the participating centers. All patients gave written informed consent before entering the study, which was performed in accordance with the Declaration of Helsinki. The study was designed by the investigators, who were also responsible for the data collection. The data analysis was performed by the investigators in conjunction with an independent statistical office.

\section{Patient Populations and Random Assignment}

Previously untreated patients with myeloma who were enrolled onto one of two studies ${ }^{16,17}$ were assessed for eligibility for the substudy. In one study, patients age $\leq 65$ years were randomly assigned to bortezomib $\left(1.3 \mathrm{mg} / \mathrm{m}^{2}\right.$ on days $1,4,8$, and 11), thalidomide (200 mg/d), and dexamethasone (320 mg) or to thalidomide and dexamethasone in each 21-day cycle for three courses as induction therapy before autologous transplantation. ${ }^{16}$ In the other study, patients age $\geq 65$ years were randomly assigned to bortezomib $\left(1.3 \mathrm{mg} / \mathrm{m}^{2}\right.$ on days $1,8,15$, and 22$)$, melphalan $\left(9 \mathrm{mg} / \mathrm{m}^{2}\right.$ on days 1 to 4$)$, prednisone (60 $\mathrm{mg} / \mathrm{m}^{2}$ on days 1 to 4$)$, and thalidomide $(50 \mathrm{mg} / \mathrm{d}$ ) for nine courses followed by continuous therapy with bortezomib $\left(1.3 \mathrm{mg} / \mathrm{m}^{2}\right.$ every 15 days $)$ and thalidomide $(50 \mathrm{mg} / \mathrm{d})$ or to bortezomib, melphalan, and prednisone for nine courses without any further continuous treatment. ${ }^{17}$ Patients randomly assigned to receive bortezomib, melphalan, and prednisone did not receive any antithrombotic prophylaxis. Patients receiving thalidomide-based regimens in both trials were eligible for the substudy. Exclusion criteria were allergy or intolerance to study drugs, clear indication or contraindication for a specific antiplatelet or anticoagulant therapy (eg, cardiac arrhythmia, cardiac ischemia, or previous history of arterial or venous thromboembolism), and active bleeding or high risk of bleeding.

A simple random assignment sequence was generated by a centralized computer. After registration in a centralized database through the Internet and validation of eligibility, patients were randomly allocated to treatments using an automated assignment procedure concealed to the investigators.

\section{Study Interventions and Clinical Follow-Up}

Patients receiving thalidomide-based regimens were randomly assigned to receive one of the following: ASA $100 \mathrm{mg} / \mathrm{d}$ orally, WAR $1.25 \mathrm{mg} / \mathrm{d}$ orally, or LMWH (enoxaparin) $40 \mathrm{mg} / \mathrm{d}$ subcutaneously. The prophylaxis was administered during the three cycles of induction therapy in the younger patients ${ }^{16}$ and during the first six cycles of induction therapy in the elderly patients. ${ }^{17}$ The antithrombotic prophylaxis was discontinued in the event of deep vein thrombosis, pulmonary embolism, arterial thrombosis, acute cardiovascular event, bleeding event, or platelet count $\leq 50,000 / \mu \mathrm{L}$. The international normalized ratio (INR) was measured on days 1 and 21 of every cycle of chemotherapy. For INR greater than 3, WAR was discontinued, and patients received adjusted doses of WAR to maintain INR less than 3. Patients attended study visits every 3 weeks during the treatment period to assess the toxicity and efficacy of treatment and subsequently at the physician's discretion to evaluate the incidence of thromboembolism in the absence of prophylaxis.

\section{Outcome Measures}

The primary end point was a composite measure defined as the proportion of patients developing a first episode of objectively confirmed symptomatic deep vein thrombosis, pulmonary embolism, arterial thrombosis, any acute cardiovascular event (acute myocardial infarction or stroke), or sudden, otherwise unexplained death (presumed to be a result of pulmonary embolism, acute myocardial infarction, or stroke) during the first 6 months from random assignment. Secondary end points included the comparison of each component of the composite primary end point, long-term cumulative incidence of the primary end point, major and minor bleeding events, and any toxicity that required interruption of study prophylaxis. All adverse events were graded according to the National Cancer Institute Common Terminology Criteria for Adverse Events (version 3). The diagnostic procedures and definitions of the conditions included in the composite end point are detailed in the Appendix (online only). Major bleeding was defined as fatal bleeding, symptomatic bleeding in a crucial area or organ, or bleeding causing a reduction in hemoglobin concentration of $\geq 2 \mathrm{~g} / \mathrm{dL}$ or necessitating transfusion of $\geq$ two units of whole blood or RBC cells. ${ }^{18}$ Minor bleeding was all other bleeding not meeting the criteria for major bleeding.

\section{Statistical Analysis}

The statistical power and the minimum effect size detectable in this trial was determined according to the sample size of the two predefined chemotherapy phase III trials, which was 450 patients for the bortezomib, thalidomide, and dexamethasone versus thalidomide and dexamethasone trial $^{16}$ and 500 patients for the bortezomib, melphalan, prednisone, and thalidomide versus bortezomib, melphalan, and prednisone trial. ${ }^{17}$ Overall, we expected approximately 700 patients treated with thalidomidecontaining regimens to be randomly assigned, with a 1:1:1 allocation ratio between the three prophylaxis regimens (approximately 230 patients per group). The main planned comparisons were ASA versus LMWH and WAR versus LMWH during the first 6 months from random assignment. The expected rate of thromboembolic events in patients with newly diagnosed myeloma treated with thalidomide-containing regimens without any prophylaxis is approximately $20 \%$ to $30 \%,{ }^{10}$ and to be considered effective, any prophylaxis should at least halve this risk. For each comparison, a sample size of 230 patients per group reaches a statistical power ranging from $53 \%$ to $86 \%$ to detect an absolute decrease of $5 \%$ to $7 \%$, respectively, between the groups, with $\alpha=.05$ (two tailed), assuming a value of $10 \%$ for the composite primary end point in the LMWH group. Because the two planned comparisons of the primary end point are true independent hypotheses, we did not adjust for multiplicity. ${ }^{19}$ Two interim analyses were planned at $15 \%$ and $45 \%$ of enrollment, using the O'Brien and Fleming group sequential test design. ${ }^{20}$

The statistic test used to compare the difference between proportions was the two-sided $z$ test, with pooled variance. A subgroup analysis of the primary composite end point was planned according to the three thalidomidecontaining regimens. To compare the incidence of the composite primary end point through the entire follow-up, taking into account the competing risk of dying from any other cause, the cumulative incidence, adjusted for competing risks, was compared between groups with the Gray's test. The hazard ratios (HRs) with 95\% CIs were estimated using the Fine and Gray's proportional hazard model. $^{21,22}$ All efficacy and safety analyses were performed according to the intent-to-treat principle and included all randomly assigned patients who received at least one dose of the study drug. Times of observation were censored on September 30, 2009. Time-to-event and continuous variables are expressed as median with interquartile range (IQR). The post hoc analysis concerning the association of thromboembolic events with potentially prognostic baseline factors (age, performance status, presence or absence of comorbidities, high International Staging System stage, dose of corticosteroids, and bortezomib association) was performed accounting for competing events. ${ }^{21,22}$ Interactions between treatment groups and covariates were assessed in the model.

\section{RESULTS}

Of 991 patients assessed for eligibility, 734 had been randomly assigned to thalidomide-containing regimes and 257 had been assigned 


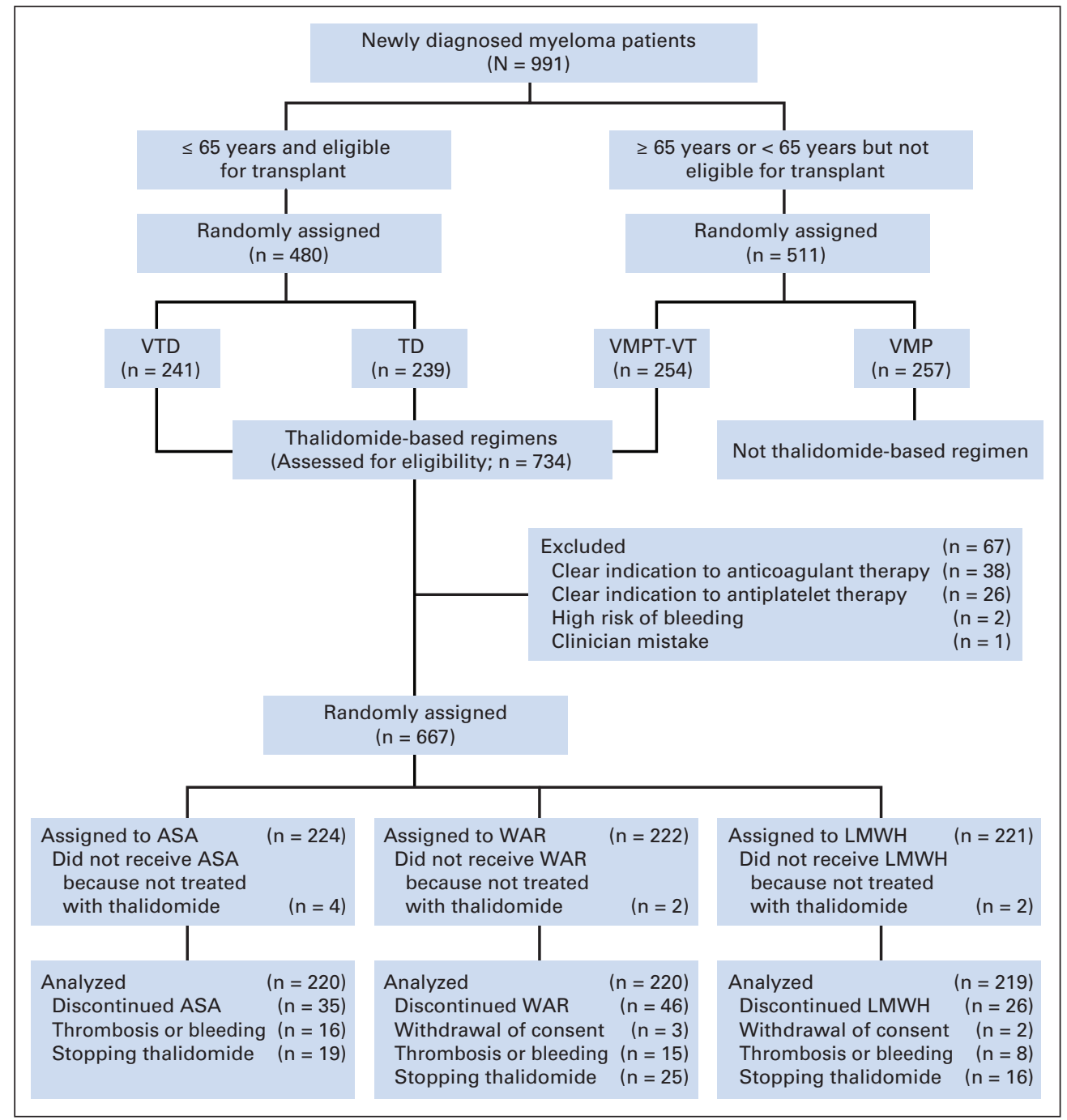

Fig 1. CONSORT diagram of patients in the trial. VTD, bortezomib, thalidomide, and dexamethasone; TD, thalidomide and dexamethasone; VMPT-VT, bortezomib, melphalan, prednisone, and thalidomide followed by continuous therapy with bortezomib and thalidomide; VMP, bortezomib, melphalan, and prednisone; ASA, aspirin; WAR, fixed low-dose warfarin; LMWH, low molecular weight heparin.

to thalidomide-free regimens (Fig 1). A total of 667 patients were enrolled onto the substudy, of whom 659 received at least one dose of the study treatment and were included in the efficacy and safety analyses (ASA, $\mathrm{n}=220$; WAR, $\mathrm{n}=220$; LMWH, $\mathrm{n}=219$; Fig 1). Sixty-seven patients were not enrolled onto the study (Fig 1). Patient characteristics were similar in all three groups (Table 1).

During the treatment period, symptomatic deep vein thrombosis, pulmonary embolism, arterial thrombosis, any acute cardiovascular event, or sudden death (composite primary end point) occurred in 14 patients $(6.4 \%)$ in the ASA group, 18 patients $(8.2 \%)$ in the WAR group, and 11 patients (5.0\%) in the LMWH group (Table 2). The absolute differences were $+1.3 \%(95 \% \mathrm{CI},-3.0 \%$ to $5.7 \% ; P=.544)$ between the ASA and LMWH groups and $+3.2 \%$ (95\% CI, $-1.5 \%$ to $7.8 \% ; P=.183$ ) between the WAR and LMWH groups (Table 3 ). The risk of composite end point was similar in patients who received ASA or LMWH and were treated with the different induction regimens (Table 4). This risk was also similar in the younger patients who received WAR or LMWH and were treated with thalidomide and dexamethasone or bortezomib, thalidomide, and dexamethasone; WAR was less effective than LMWH in the elderly patients treated with bortezomib, melphalan, prednisone, and thalidomide (absolute difference, $+11.3 \%$; $95 \%$ CI, $3.4 \%$ to $19.2 \% ; P=.006$; Table 4$)$. The incidence of thromboembolic events, acute cardiovascular events, and sudden deaths was $5.4 \%$ in patients receiving thalidomide and bortezomib and $7.2 \%$ in patients receiving thalidomide without bortezomib $(P=.60)$. In the thalidomide-bortezomib patients, the absolute differences were $+1.5 \%(95 \% \mathrm{CI},-3.9 \%$ to $7.1 \%$; $P=.56)$ between the ASA and LMWH groups and $+4.4 \%$ (95\% CI, $-1.5 \%$ to $10.7 \% ; P=.13$ ) between the WAR and LMWH groups. In the thalidomide patients, the absolute differences were $+1.0 \%$ (95\% CI, $-7.9 \%$ to $9.8 \% ; P=.81$ ) between the ASA and LMWH groups and $+0.8 \%$ $(95 \% \mathrm{CI},-8.0 \%$ to $9.5 \% ; P=.84)$ between the WAR and LMWH groups.

The most frequent complications were thromboembolic events; these occurred in 13 patients (5.9\%) in the ASA group, 18 patients $(8.2 \%)$ in the WAR group, and seven patients $(3.2 \%)$ in the LMWH group in the first 6 months (Table 2). Symptomatic pulmonary embolism occurred in eight patients in the first 6 months, including four patients in the ASA group (one of whom died) and four patients in the WAR group. No pulmonary embolism was reported in the LMWH group. The absolute differences for serious thromboembolic events were $+2.7 \%(95 \% \mathrm{CI},-1.2 \%$ to $6.6 \% ; P=.173)$ between the ASA 


\begin{tabular}{|c|c|c|c|c|c|c|}
\hline \multirow[b]{2}{*}{$\begin{array}{c}\text { Demographic or Clinical } \\
\text { Characteristic }\end{array}$} & \multicolumn{2}{|c|}{ ASA $(n=220)$} & \multicolumn{2}{|c|}{ WAR $(n=220)$} & \multicolumn{2}{|c|}{ LMWH $(n=219)$} \\
\hline & $\begin{array}{l}\text { No. of } \\
\text { Patients }\end{array}$ & $\%$ & $\begin{array}{c}\text { No. of } \\
\text { Patients }\end{array}$ & $\%$ & $\begin{array}{c}\text { No. of } \\
\text { Patients }\end{array}$ & $\%$ \\
\hline \multicolumn{7}{|l|}{ Age, years } \\
\hline Median & \multicolumn{2}{|c|}{61} & \multicolumn{2}{|c|}{60} & \multicolumn{2}{|c|}{62} \\
\hline IQR & \multicolumn{2}{|c|}{$55-66$} & \multicolumn{2}{|c|}{$54-66$} & \multicolumn{2}{|c|}{$55-66$} \\
\hline$\leq 55$ & 54 & 25 & 61 & 28 & 55 & 25 \\
\hline $56-65$ & 103 & 47 & 100 & 45 & 102 & 47 \\
\hline $66-75$ & 52 & 24 & 43 & 20 & 50 & 23 \\
\hline$>75$ & 11 & 5 & 16 & 7 & 12 & 5 \\
\hline Male & 117 & 53 & 115 & 52 & 130 & 59 \\
\hline \multicolumn{7}{|l|}{$\mathrm{MM}$ treatment } \\
\hline TD & 79 & 36 & 81 & 37 & 76 & 35 \\
\hline VTD & 78 & 35 & 77 & 35 & 79 & 36 \\
\hline VMPT-VT & 63 & 29 & 62 & 28 & 64 & 29 \\
\hline \multicolumn{7}{|l|}{ ISS } \\
\hline I & 87 & 40 & 84 & 38 & 86 & 39 \\
\hline II & 87 & 40 & 86 & 39 & 80 & 37 \\
\hline III & 34 & 15 & 38 & 17 & 40 & 18 \\
\hline Data missing & 12 & 5 & 12 & 5 & 13 & 6 \\
\hline \multicolumn{7}{|l|}{ Creatinine, mg/dL* } \\
\hline Median & \multirow{2}{*}{\multicolumn{2}{|c|}{$\begin{array}{c}0.98 \\
0.8-1.19\end{array}$}} & \multirow{2}{*}{\multicolumn{2}{|c|}{$\begin{array}{c}1.0 \\
0.8-1.19\end{array}$}} & \multirow{2}{*}{\multicolumn{2}{|c|}{$\begin{array}{c}0.96 \\
0.8-1.1\end{array}$}} \\
\hline IQR & & & & & & \\
\hline \multicolumn{7}{|l|}{ Glycemia, mg/dL* } \\
\hline Median & \multicolumn{2}{|c|}{94} & \multicolumn{2}{|c|}{92} & \multicolumn{2}{|c|}{93} \\
\hline IQR & \multicolumn{2}{|c|}{$86-102$} & \multicolumn{2}{|c|}{$85-102$} & \multicolumn{2}{|c|}{$86-102$} \\
\hline Data missing & 13 & 6 & 12 & 5 & 19 & 7 \\
\hline \multicolumn{7}{|l|}{ Karnofsky performance status } \\
\hline$\leq 70 \%$ & 54 & 25 & 64 & 29 & 65 & 30 \\
\hline Data missing & 8 & 4 & 12 & 5 & 8 & 4 \\
\hline Diabetes & 10 & 5 & 9 & 4 & 8 & 4 \\
\hline Cardiovascular disease & 35 & 16 & 48 & 22 & 38 & 17 \\
\hline Hypertension & 29 & 13 & 44 & 20 & 33 & 15 \\
\hline Acute myocardial infarction & 0 & 0 & 1 & 0.5 & 2 & 1 \\
\hline Heart failure & 0 & 0 & 1 & 0.5 & 0 & 0 \\
\hline Arrhythmia & 7 & 3 & 3 & 1 & 4 & 2 \\
\hline Other & 0 & 0 & 0 & 0 & 1 & 0.5 \\
\hline Orthopedic disease & 0 & 0 & 2 & 1 & 1 & 0.5 \\
\hline Dyslipidemia & 4 & 2 & 7 & 3 & 4 & 2 \\
\hline Prior thromboembolism & 2 & 1 & 2 & 1 & 1 & 0.5 \\
\hline$>$ two comorbidities & 7 & 3 & 10 & 5 & 4 & 2 \\
\hline
\end{tabular}

and LMWH groups and $+5.0 \%$ (95\% CI, $0.7 \%$ to $9.3 \% ; P=.024)$ between the WAR and LMWH groups (Table 3 ). The regression model showed a tendency toward a higher risk of developing thromboembolic events in the following patients: patients older than age 60 years (HR, $1.75 ; 95 \% \mathrm{CI}, 0.84$ to 3.62 ); patients with more than two comorbidities (HR, 1.43; 95\% CI, 0.33 to 6.25); patients with a Karnofsky performance status less than $70 \%(\mathrm{HR}, 1.47 ; 95 \% \mathrm{CI}, 0.70$ to 3.09); patients not receiving bortezomib ( $\mathrm{HR}, 1.38$; 95\% CI, 0.66 to 2.89 ); and patients receiving higher doses of dexamethasone ( $\mathrm{HR}$, 1.97; 95\% CI, 0.72 to 5.39 ).

After a median follow-up of 24.9 months (IQR, 18.4 to 32.0 months), 58 patients ( $8.8 \%$ ) experienced one of the events included in the composite primary end point (Table 2). The cumulative proportions of thromboembolic events, acute cardiovascular events, and sudden deaths adjusted for competing risks at 18 months were 0.08 ( $95 \%$ CI, 0.05 to 0.12 ) in the ASA group, 0.10 (95\% CI, 0.06 to 0.14 ) in the WAR group, and 0.08 (95\% CI, 0.04 to 0.11$)$ in the LMWH group $(P=.69$; Fig 2$)$. The HRs for ASA and WAR versus LMWH, adjusted for competing risks, were 1.13 (95\% CI, 0.59 to $2.17 ; P=.716)$ and 1.31 ( $95 \% \mathrm{CI}, 0.70$ to $2.47 ; P=.397$ ), respectively. Any grade 3 or 4 thromboembolic events were reported in 17 patients $(7.7 \%)$ in the ASA group, 21 patients (9.5\%) in the WAR group, and 11 patients $(5.0 \%)$ in the LMWH group (Table 2 ). No late pulmonary embolism was observed. Most grade 3 to 4 thromboembolic events (78\%) occurred within the first 4 months; the median times to onset were 2.3 months (IQR, 1.4 to 3.1 months) in the ASA group, 2.3 months (IQR, 1.4 to 3.5 months) in the WAR group, and 4.5 months (IQR, 1.4 to 11.1 months) in the LMWH group. Overall, one patient died in the 


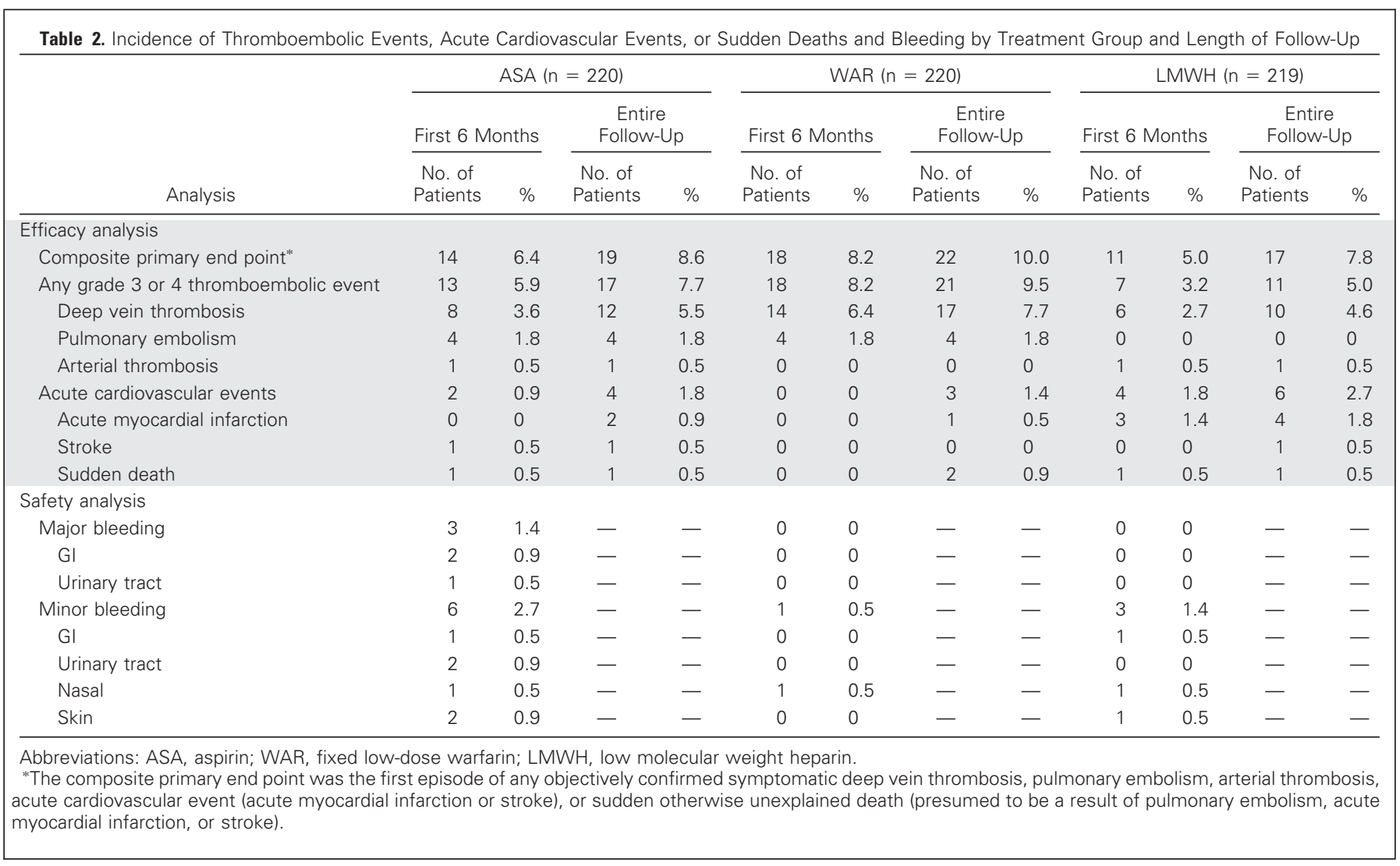

ASA group (pulmonary embolism), two patients died in the WAR group (acute myocardial infarction and cardiac arrest), and one patient died in the LMWH group (cardiac arrest; Table 2).

The median durations of prophylaxis were 2.6 months (IQR, 2.1 to 4.0 months) in the ASA group, 2.4 months (IQR, 2.1 to 3.5 months) in the WAR group, and 2.6 months (IQR, 2.1 to 4.5 months) in the LMWH group. Thirty-five patients (16\%) in the ASA group, 46 patients (21\%) in the WAR group, and 26 patients $(12 \%)$ in the LMWH group discontinued prophylaxis prematurely, mainly because of thromboembolic, cardiovascular, or bleeding events, or because of thalidomide discontinuation for adverse events or progressive disease (Fig 1). There were no statistically or clinically significant differences among the three groups in terms of the incidences of any adverse events during the treat- ment or follow-up periods. Major bleeding occurred during the first 6 months in three patients $(1.4 \%)$ who received ASA but in no patients who received either WAR or LMWH. Six patients $(2.7 \%)$ in the ASA group, one patient $(0.5 \%)$ in the WAR group, and three patients $(1.4 \%)$ in the LMWH group had minor bleeding (Table 2).

\section{DISCUSSION}

Our findings showed that, in patients with myeloma treated with thalidomide, the incidence of symptomatic deep vein thrombosis, pulmonary embolism, arterial thrombosis, any acute cardiovascular event, or sudden death was $6 \%$ during ASA, $8 \%$ during WAR, and 5\% during LMWH thromboprophylaxis, without statistically significant

Table 3. Absolute Risk Difference of Thromboembolic Events, Acute Cardiovascular Events, or Sudden Deaths and Bleeding During the First 6 Months for ASA and WAR Compared With LMWH

\begin{tabular}{|c|c|c|c|c|c|c|}
\hline \multirow[b]{2}{*}{ Event } & \multicolumn{3}{|c|}{ ASA $v$ LMWH } & \multicolumn{3}{|c|}{ WAR $v$ LMWH } \\
\hline & $\begin{array}{c}\text { Absolute } \\
\text { Difference (\%) }\end{array}$ & $95 \% \mathrm{Cl}(\%)$ & $P$ & $\begin{array}{c}\text { Absolute } \\
\text { Difference (\%) }\end{array}$ & $95 \% \mathrm{Cl}(\%)$ & $P$ \\
\hline Composite primary end point & 1.3 & -3.0 to 5.7 & .544 & 3.2 & -1.5 to 7.8 & .183 \\
\hline Any grade 3 or 4 thromboembolic event & 2.7 & -1.2 to 6.6 & .173 & 5.0 & 0.7 to 9.3 & .024 \\
\hline Acute cardiovascular events & -0.9 & -2.7 to 0.9 & .313 & -1.4 & -2.9 to 0.2 & .082 \\
\hline Major bleeding & 1.4 & -0.2 to 2.9 & .083 & 0.0 & -1.7 to 1.7 & 1.000 \\
\hline Minor bleeding & 1.3 & -1.3 to 4 & .316 & -0.9 & -2.7 to 0.9 & .313 \\
\hline
\end{tabular}

Abbreviations: ASA, aspirin; WAR, fixed low-dose warfarin; LMWH, low molecular weight heparin. 
Table 4. Absolute Risk Difference of Thromboembolic Events, Acute Cardiovascular Events, or Sudden Deaths During the First 6 Months of Treatment for ASA and WAR Compared With LMWH, Stratified by Myeloma Treatment

\begin{tabular}{|c|c|c|c|c|c|c|}
\hline \multirow[b]{2}{*}{ Treatment } & \multicolumn{3}{|c|}{ ASA $v$ LMWH (heterogeneity test, $P=$.748) } & \multicolumn{3}{|c|}{ WAR $v$ LMWH (heterogeneity test, $P=.077$ ) } \\
\hline & $\begin{array}{c}\text { Absolute } \\
\text { Difference (\%) }\end{array}$ & $95 \% \mathrm{Cl}(\%)$ & $P$ & $\begin{array}{c}\text { Absolute } \\
\text { Difference (\%) }\end{array}$ & $95 \% \mathrm{Cl}(\%)$ & $P$ \\
\hline TD & 1.0 & -7.1 to 9.1 & .805 & 0.8 & -7.1 to 8.8 & .839 \\
\hline VTD & 0.1 & -8.2 to 8.4 & .982 & -1.1 & -9.1 to 6.9 & .788 \\
\hline VMPT-VT & 3.2 & -1.2 to 7.5 & .151 & 11.3 & 3.4 to 19.2 & .006 \\
\hline
\end{tabular}

Abbreviations: ASA, aspirin; WAR, fixed low-dose warfarin; LMWH, low molecular weight heparin; TD, thalidomide and dexamethasone; VTD, bortezomib, thalidomide, and dexamethasone; VMPT-VT, bortezomib, melphalan, prednisone, and thalidomide followed by continuous therapy with bortezomib and thalidomide.

differences between the ASA or WAR groups compared with the LMWH group.

Without thromboprophylaxis, the incidence of thromboembolic events has been reported to be $14 \%$ to $26 \%$ in patients with myeloma receiving thalidomide plus dexamethasone $e^{23,24}$ and $10 \%$ to $20 \%$ in patients receiving thalidomide plus melphalan. ${ }^{23}$ In the ASA group, the incidence of thromboembolism was $6 \%$ over 6 months. In a previous study, the rate of thromboembolism with ASA prophylaxis was reported to be $11 \%$ to $18 \% .{ }^{14}$ In our study, $8 \%$ of patients had thromboembolic events during WAR therapy (6 months). In other studies of patients taking thalidomide, dexamethasone, and fixed lowdose WAR, the rate of thromboembolism has been reported to be $13 \%$ to $25 \% .{ }^{11,12,25}$ In our LMWH group, the incidence of thromboembolism was 3\% (6 months), which is consistent with other trials including thalidomide plus LMWH (along with various combinations of dexamethasone, prednisone, and melphalan), where the rate of thromboembolism has been reported to be $0 \%$ to $9 \% .^{5,12,26}$ In our study, the risk of thromboembolism was 1.38 times higher among

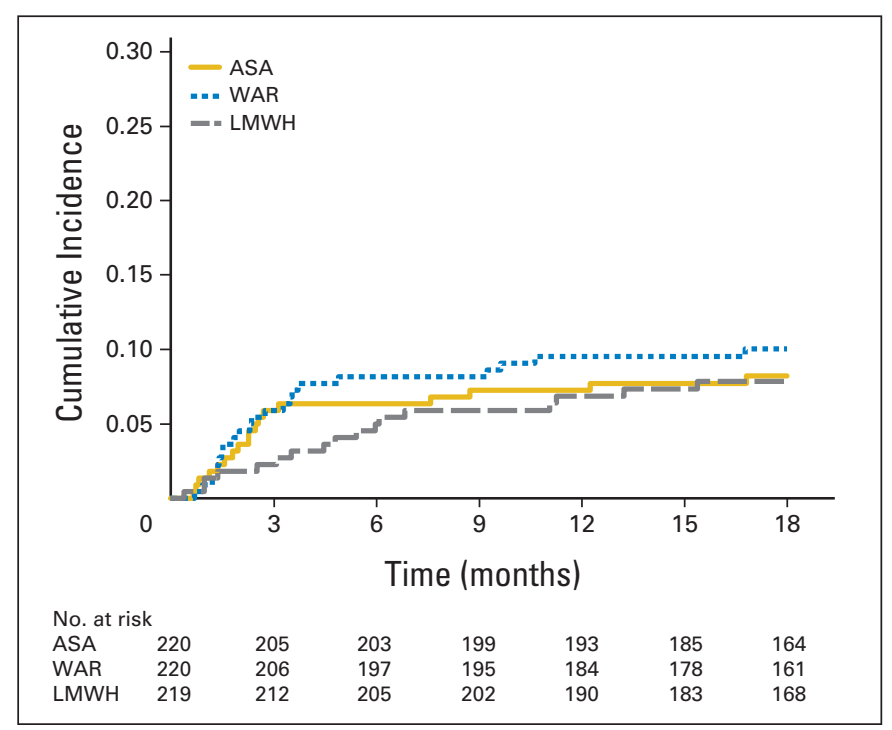

Fig 2. Cumulative incidence (proportion) of the primary composite end point, adjusted for competing risks (other causes of death) by treatment group. The follow-up end was defined at 18 months because no additional events occurred after this time. The cumulative incidences of the primary composite end point (thromboembolic events, acute cardiovascular events, or sudden deaths), adjusted for competing risks, at 18 months were $0.08(95 \% \mathrm{Cl}, 0.05$ to 0.12$)$ in the aspirin (ASA) group, $0.10(95 \% \mathrm{Cl}, 0.06$ to 0.14$)$ in the fixed low-dose warfarin (WAR) group, and $0.08(95 \% \mathrm{Cl}, 0.04$ to 0.11$)$ in the low molecular weight heparin (LMWH) group (Gray's test $P=.69$ ). patients receiving thalidomide without bortezomib, which may support the protective role of bortezomib against thromboembolism. Bortezomib alone or in combination with dexamethasone or chemotherapy did not increase the risk of thromboembolism, ${ }^{27-29}$ whereas it seems to confer protection when administered with thalidomide. ${ }^{16,30}$ The thalidomide analog lenalidomide is emerging as an interesting alternative in the first-line therapy of myeloma. Most recent trials have included thromboprophylaxis with ASA, which seemed to be effective. ${ }^{31,32}$ The combination of lenalidomide-bortezomibdexamethasone with ASA prophylaxis showed promising results, with a severe thromboembolism incidence of $5 \% .{ }^{33}$

Prevention of thromboembolic complications has a central role in the treatment of patients with cancer because the occurrence of thromboembolism may cause chemotherapy discontinuation, increases health expenditure, and requires anticoagulant treatment with a higher risk of complications. To date, only results from small phase II studies on single antithrombotic agents including ASA, WAR, or LMWH are available. ${ }^{11-14,23,24,26}$ To our knowledge, our study is the first randomized trial to question which antithrombotic prophylaxis may be more effective. All three regimens were equally effective and safe in patients with standard risk of thromboembolism. LMWH was slightly superior to ASA and WAR, but some practical issues, such as costs and feasibility of self-injection, should be considered. Both ASA and WAR may be considered valid alternative options and are less expensive, are administered orally, and do not require constant monitoring.

In a recent meta-analysis, when thalidomide was used as maintenance after autologous transplantation, the incidence of thromboembolic events was $4 \%$ to $6 \%$, and the risk of thromboembolism was 1.95 times higher than in patients who did not receive thalidomide. ${ }^{10}$ The use of ASA for a longer period of time may reduce the risk of late thromboembolism, and future studies should address this question.

The rates of pulmonary embolism and bleeding were higher among patients receiving ASA than patients receiving LMWH. The risk of bleeding in the ASA group was comparable with that seen in other studies on thromboprophylaxis in patients with cancer. ${ }^{34,35}$ If these increased risks are confirmed in a larger series of patients, they may outweigh the practical advantages of ASA versus LMWH.

Patients at high risk of thromboembolic events, such as patients with previous history of thromboembolism, severe cardiac disease, uncontrolled diabetes, infections, immobilization, or surgery, were not included in our analysis because they had a clear indication of anticoagulant or antiplatelet therapy. This is a major limitation of the 
study. LMWH prophylaxis should remain mandatory in patients at high risk of venous thromboembolism for at least the first 6 months of therapy; thereafter, ASA may be considered to reduce the occurrence of late thromboembolic events when long-term thalidomide therapy is planned.

Further limitations of our study are the absence of a placebo group and the open-label design. However, the inclusion of a placebo arm would not have been ethical because all patients enrolled onto this study were treated with thalidomide-containing regimens and could have an increased risk of thromboembolic events.

To conclude, both ASA and WAR showed similar safety and efficacy in reducing thromboembolic complications when compared with LMWH in patients with myeloma treated with thalidomidebased regimens. Antiangiogenic agents may inhibit the healing of chemotherapy-induced endothelial injury, increasing the risk of thromboembolism. ${ }^{36}$ Of note, defibrotide, which targets endothelial cell damage, may have a protective effect against thromboembolism, specifically induced by thalidomide. ${ }^{37,38}$ New and effective antithrombotic agents with reduced drug interactions and without the need for constant monitoring could optimize patient care and eventually modify the indication for use and duration of thromboprophylaxis.

\section{AUTHORS' DISCLOSURES OF POTENTIAL CONFLICTS OF INTEREST}

Although all authors completed the disclosure declaration, the following author (s) indicated a financial or other interest that is relevant to the subject matter under consideration in this article. Certain relationships marked with a " $U$ " are those for which no compensation was received; those relationships marked with a " $C$ " were compensated. For a detailed description of the disclosure categories, or for more information about ASCO's conflict of interest policy, please refer to the Author Disclosure Declaration and the Disclosures of Potential Conflicts of Interest section in Information for Contributors.

Employment or Leadership Position: None Consultant or Advisory Role: Antonio Palumbo, Celgene (C), Janssen-Cilag (C); Michele Cavo,
Janssen-Cilag (C), Millennium Pharmaceuticals (C); Sara Bringhen, Merck Sharp \& Dohme (C); Tommaso Caravita, Celgene (C), Johnson \& Johnson (C); Mario Boccadoro, Celgene (C), Janssen-Cilag (C) Stock Ownership: None Honoraria: Antonio Palumbo, Celgene, Janssen-Cilag; Michele Cavo, Janssen-Cilag, Novartis; Sara Bringhen, Celgene, Janssen-Cilag, Novartis; Francesca Patriarca, Celgene, Janssen-Cilag, Schering-Plough; Roberto Ria, Novartis, Celgene, Janssen-Cilag; Luca Baldini, Celgene, Janssen-Cilag; Francesca Elice, Celgene; Massimo Offidani, Celgene, Janssen-Cilag; Mario Boccadoro, Celgene, Janssen-Cilag Research Funding: Roberto Marasca, Celgene; Tommaso Caravita, Celgene, Johnson \& Johnson; Mario Boccadoro, Celgene, Janssen-Cilag Expert Testimony: None Other Remuneration: None

\section{AUTHOR CONTRIBUTIONS}

Conception and design: Antonio Palumbo, Michele Cavo,

Mario Boccadoro

Provision of study materials or patients: Sara Bringhen, Elena Zamagni, Alessandra Romano, Francesca Patriarca, Davide Rossi, Fabiana Gentilini, Claudia Crippa, Monica Galli, Chiara Nozzoli, Roberto Ria, Roberto Marasca, Vittorio Montefusco, Luca Baldini, Francesca Elice, Vincenzo Callea, Stefano Pulini, Angelo M. Carella, Renato Zambello, Giulia Benevolo, Valeria Magarotto, Paola Tacchetti, Norbert Pescosta, Claudia Cellini, Claudia Polloni, Tommaso Caravita, Fortunato Morabito, Massimo Offidani, Patrizia Tosi

Data analysis and interpretation: Antonio Palumbo, Michele Cavo, Sara Bringhen, Andrea Evangelista

Manuscript writing: Antonio Palumbo, Michele Cavo, Sara Bringhen, Mario Boccadoro

Final approval of manuscript: Antonio Palumbo, Michele Cavo, Sara Bringhen, Elena Zamagni, Alessandra Romano, Francesca Patriarca, Roberto Ria, Davide Rossi, Fabiana Gentilini, Claudia Crippa, Monica Galli, Chiara Nozzoli, Roberto Ria, Roberto Marasca, Vittorio Montefusco, Luca Baldini, Francesca Elice, Vincenzo Callea, Stefano Pulini, Angelo M. Carella, Renato Zambello, Giulia Benevolo, Valeria Magarotto, Paola Tacchetti, Norbert Pescosta, Claudia Cellini, Claudia Polloni, Andrea Evangelista, Tommaso Caravita, Fortunato Morabito, Massimo Offidani, Patrizia Tosi, Mario Boccadoro

\section{REFERENCES}

1. Sallah S, Wan JY, Nguyen NP: Venous thrombosis in patients with solid tumors: Determination of frequency and characteristics. Thromb Haemost 87: 575-579, 2002

2. Walsh-McMonagle D, Green D: Lowmolecular-weight heparin in the management of Trousseau's syndrome. Cancer 80:649-655, 1997

3. Blom JW, Doggen CJ, Osanto S, et al: Malignancies, prothrombotic mutations, and the risk of venous thrombosis. JAMA 293:715-722, 2005

4. Bick RL: Cancer-associated thrombosis. N Engl J Med 349:109-111, 2003

5. Palumbo A, Bringhen S, Caravita $T$, et al: Oral melphalan and prednisone chemotherapy plus thalidomide compared with melphalan and prednisone alone in elderly patients with multiple myeloma: Randomised controlled trial. Lancet 367:825-831, 2006

6. Facon T, Mary JY, Hulin C, et al: Melphalan and prednisone plus thalidomide versus melphalan and prednisone alone or reduced-intensity autologous stem cell transplantation in elderly patients with multiple myeloma (IFM 99-06): A randomised trial. Lancet 370:1209-1218, 2007
7. Hulin $C$, Facon $T$, Rodon $P$, et al: Efficacy of melphalan and prednisone plus thalidomide in patients older than 75 years with newly diagnosed multiple myeloma: IFM 01/01 trial. J Clin Oncol 27:3664-3670, 2009

8. Musallam KM, Dahdaleh FS, Shamseddine $\mathrm{Al}$, et al: Incidence and prophylaxis of venous thromboembolic events in multiple myeloma patients receiving immunomodulatory therapy. Thromb Res 123:679-686, 2009

9. El Accaoui RN, Shamseddeen WA, Taher AT: Thalidomide and thrombosis: A meta-analysis. Thromb Haemost 97:1031-1036, 2007

10. Hicks LK, Haynes AE, Reece DE, et al: A meta-analysis and systematic review of thalidomide for patients with previously untreated multiple myeloma. Cancer Treat Rev 34:442-452, 2008

11. Cavo M, Zamagni E, Tosi $P$, et al: Superiority of thalidomide and dexamethasone over vincristinedoxorubicin-dexamethasone (VAD) as primary therapy in preparation for autologous transplantation for multiple myeloma. Blood 106:35-39, 2005

12. Weber D, Rankin K, Gavino M, et al: Thalidomide alone or with dexamethasone for previously untreated multiple myeloma. J Clin Oncol 21:16-19, 2003
13. Zangari $M$, Barlogie $B$, Anaissie $E$, et al: Deep vein thrombosis in patients with multiple myeloma treated with thalidomide and chemotherapy: Effects of prophylactic and therapeutic anticoagulation. Br J Haematol 126:715-721, 2004

14. Baz R, Li L, Kottke-Marchant K, et al: The role of aspirin in the prevention of thrombotic complications of thalidomide and anthracycline-based chemotherapy for multiple myeloma. Mayo Clin Proc 80:1568-1574, 2005

15. Lyman $\mathrm{GH}$, Khorana $A A$, Falanga $A$, et al: American Society of Clinical Oncology guideline: Recommendations for venous thromboembolism prophylaxis and treatment in patients with cancer. J Clin Oncol 25:5490-5505, 2007

16. Cavo M, Tacchetti P, Patriarca F, et al: Bortezomib with thalidomide plus dexamethasone compared with thalidomide plus dexamethasone as induction therapy before, and consolidation therapy after, double autologous stem-cell transplantation in newly diagnosed multiple myeloma: A randomised phase 3 study. Lancet 376:20752085, 2010

17. Palumbo A, Bringhen $S$, Rossi D, et al: Bortezomib-melphalan-prednisone-thalidomide followed by maintenance with bortezomib-thalidomide compared with bortezomib-melphalan-prednisone for initial 
treatment of multiple myeloma: A randomised controlled trial. J Clin Oncol 28:5101-5109, 2010

18. Schulman S, Kearon C, Subcommittee on Control of Anticoagulation of the Scientific and Standardization Committee of the International Society on Thrombosis and Haemostasis: Definition of major bleeding in clinical investigations of antihemostatic medicinal products in non-surgical patients. J Thromb Haemost 3:692-694, 2005

19. Proschan MA, Waclawiw MA: Practical guidelines for multiplicity adjustment in clinical trials. Control Clin Trials 21:527-539, 2000

20. Gooley TA, Leisenring W, Crowley J, et al: Estimation of failure probabilities in the presence of competing risks: New representations of old estimators. Stat Med 18:695-706, 1999

21. Gray RJ: A class of K-sample tests for comparing the cumulative incidence of a competing risk. Ann Stat 16:1141-1154, 1988

22. Fine $P$, Gray R: A proportional hazards model for the subdistribution of a competing risk. J Am Stat Assoc 94:496-506, 1999

23. Palumbo A, Rajkumar SV, Dimopoulos MA, et al: Prevention of thalidomide- and lenalidomideassociated thrombosis in myeloma. Leukemia 22: 414-423, 2008

24. Cavo M, Zamagni E, Cellini C, et al: Deep-vein thrombosis in patients with multiple myeloma receiving first-line thalidomide-dexamethasone therapy. Blood 100:2272-2273, 2002

25. Miller KC, Padmanabhan S, Dimicelli L, et al: Prospective evaluation of low-dose warfarin for pre- vention of thalidomide associated venous thromboembolism. Leuk Lymphoma 47:2339-2343, 2006

26. Ludwig H, Hajek R, Tóthová $E$, et al: Thalidomide-dexamethasone compared with melphalanprednisolone in elderly patients with multiple myeloma. Blood 113:3435-3442, 2009

27. Harousseau JL, Attal M, Avet-Loiseau H, et al: Bortezomib plus dexamethasone is superior to vincristine plus doxorubicin plus dexamethasone as induction treatment prior to autologous stem-cell transplantation in newly diagnosed multiple myeloma: Results of the IFM 2005-01 phase III trial. J Clin Oncol 28:4621-4629, 2010

28. Jagannath $S$, Durie BG, Wolf JL, et al: Extended follow-up of a phase 2 trial of bortezomib alone and in combination with dexamethasone for the frontline treatment of multiple myeloma. $\mathrm{Br} J$ Haematol 146:619-626, 2009

29. Richardson PG, Xie W, Mitsiades C, et al: Single-agent bortezomib in previously untreated multiple myeloma: Efficacy, characterization of peripheral neuropathy and molecular correlation with response and neuropathy. J Clin Oncol 27:35183525, 2009

30. Zangari M, Guerrero J, Cavallo F, et al: Hemostatic effects of bortezomib treatment in patients with relapsed or refractory multiple myeloma. Haematologica 93:953-954, 2008

31. Palumbo A, Falco $P$, Corradini $P$, et al: Melphalan, prednisone, and lenalidomide treatment for newly diagnosed myeloma: A report from the GIMEMAItalian Multiple Myeloma Network. J Clin Oncol 25:4459-4465, 2007
32. Palumbo A, Dimopoulos MA, Delforge $M$, et al: A phase III study to determine the efficacy and safety of lenalidomide in combination with melphalan and prednisone (MPR) in elderly patients with newly diagnosed multiple myeloma. Blood 114:253, 2009 (abstr 613)

33. Richardson PG, Weller $E$, Lonial $S$, et al: Lenalidomide, bortezomib, and dexamethasone combination therapy in patients with newly diagnosed multiple myeloma. Blood 116:679-686, 2010

34. Agnelli G, Gussoni G, Bianchini C, et al: Nadroparin for the prevention of thromboembolic events in ambulatory patients with metastatic or locally advanced solid cancer receiving chemotherapy: A randomised, placebo-controlled, double-blind study. Lancet Oncol 10:943-949, 2009

35. Levine M, Hirsh J, Gent M, et al: Double-blind randomised trial of a very-low-dose warfarin for prevention of thromboembolism in stage IV breast cancer. Lancet 343:886-889, 1994

36. Kuenen BC: Analysis of prothrombotic mechanisms and endothelial perturbation during treatment with angiogenesis inhibitors. Pathophysiol Haemost Thromb 33:13-14, 2002 (suppl 1)

37. Echart C, Disasto M, Ferro L, et al: Defibrotide induces downregulation of heparenase expression in multiple myeloma cell lines: A novel mechanism of action. Haematologica 92:146, 2007 (suppl 2)

38. Palumbo A, Larocca A, Genuardi $M$, et al: Melphalan, prednisone, thalidomide and defibrotide in relapsed/refractory multiple myeloma: Results of a multicenter phase I/II trial. Haematologica 95: 1144-1149, 2010 